\title{
Importance économique des produits forestiers non ligneux dans quelques villages du Sud-Cameroun
}

\section{Guillaume LESCUYER}

\section{Cirad-Cifor}

URP Bsef

Biens et services des écosystèmes forestiers tropicaux :

l'enjeu du changement global

\section{BP 22572}

Yaoundé

Cameroun

\section{Cirad}

\section{URP Bsef}

Biens et services des écosystèmes forestiers tropicaux :

l'enjeu du changement global

TA C-105 / D Campus international de Baillarguet

34398 Montpellier Cedex 5

France

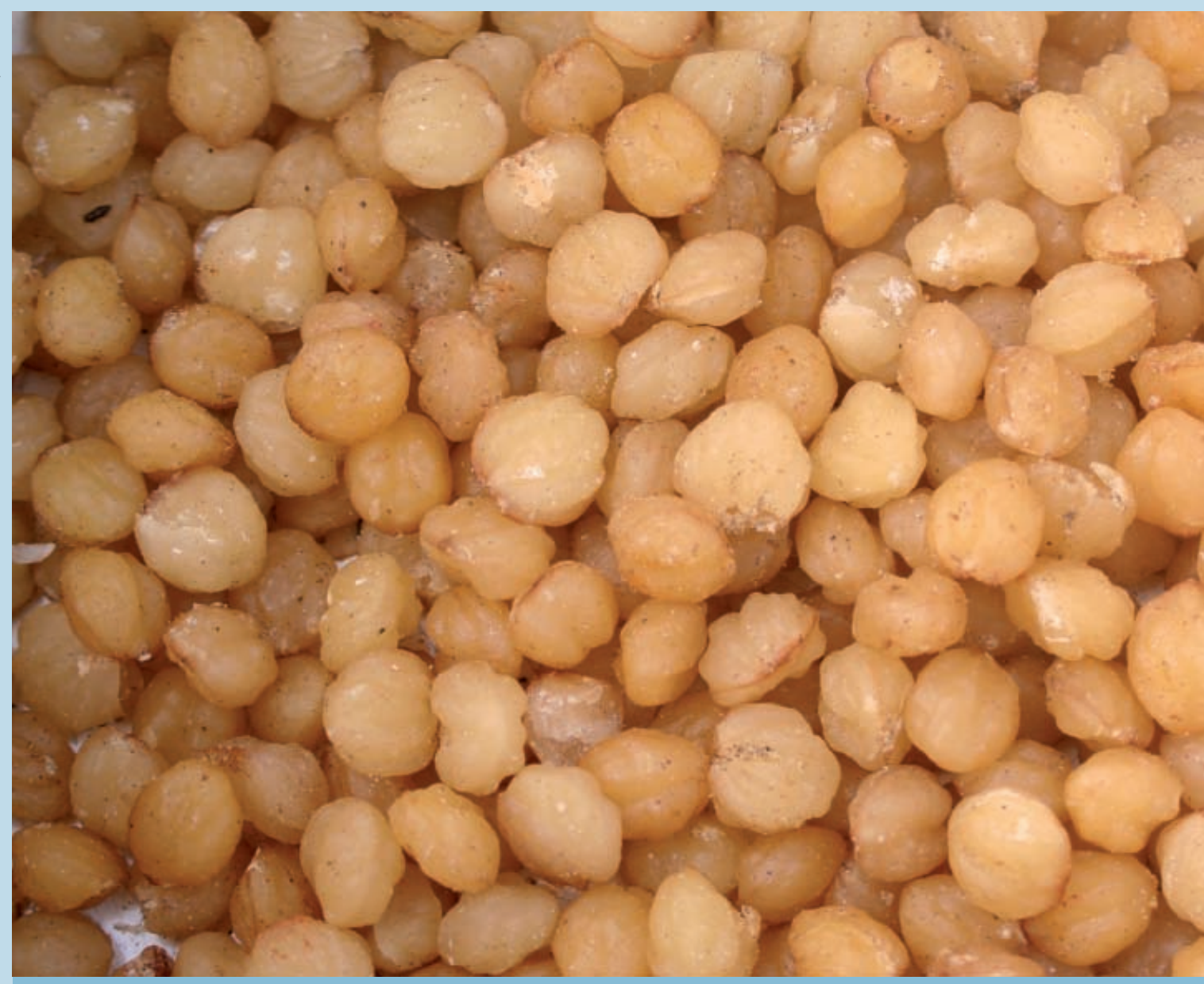

Photo 1.

La noix du njansang, Ricinodendron heudelotii.

Photo G. Lescuyer. 


\section{RÉSUMÉ}

\section{IMPORTANCE ÉCONOMIQUE DES PRODUITS FORESTIERS NON LIGNEUX DANS QUELQUES VILLAGES DU SUD- CAMEROUN}

De nombreuses organisations font la promotion des produits forestiers non ligneux (Pfnl) en Afrique centrale afin d'améliorer la gestion de la forêt et de contribuer à l'élévation des niveaux de vie en milieu rural. Trois sites ont été retenus dans la zone méridionale du Cameroun pour analyser l'impact réel de ces filières de Pfnl sur l'économie des foyers au milieu des années 1990 puis en 2008-2009. Sur la base d'un suivi annuel des pratiques forestières et des budgets des ménages, l'article montre que les Pfnl restent encore une ressource finacièrement marginale pour les foyers de ces zones forestières. Si presque tous les foyers pratiquent effectivement la cueillette, très peu sont impliqués dans une véritable valorisation commerciale de ces produits. Leur vente ne représente que quelques pourcents de leur revenu total, bien loin derrière l'agriculture. Dans un tel contexte où les Pfnl demeurent un simple moyen de subsistance, une stratégie de promotion de ces produits devra analyser en détail les arbitrages économiques et les contraintes sociales auxquels sont confrontés ces ménages pour espérer être efficace et pertinente.

Mots-clés : produits forestiers non ligneux, économie, Cameroun.

\section{ABSTRACT}

\section{THE ECONOMIC IMPORTANCE OF NON- TIMBER FOREST PRODUCTS - VILLAGE CASE STUDIES IN SOUTHERN CAMEROON}

Many different organisations are working to promote non-timber forest products (NTFP) in central Africa, in order to improve forest management and help to raise living standards in rural areas. Three sites in Southern Cameroon were chosen for this study designed to analyse the true impact of NTFP sectors on the household economy, in the mid-1990s and then in 20082009. This article shows that, based on annual monitoring of forestry practices and household budgets, NTFPs are still an financially marginal resource for families in these forest areas. Virtually all households gather products from the wild but very few are involved in actual commercial marketing of these products, whose sales contribute a small proportion of their total income, much less than farming. In a context where NTFPs are used for subsistence needs only, any strategy to promote them, if it is to be effective and relevant, must make an in-depth analysis of the economic trade-offs and social constraints weighing on these households.

Keywords: non-timber forest products, economy, Cameroon.

\section{RESUMEN}

\section{IMPORTANCIA ECONÓMICA DE LOS PRODUCTOS FORESTALES NO MADEREROS EN ALGUNOS PUEBLOS DEL SUR DE CAMERÚN}

Numerosas organizaciones efectúan la promoción de los productos forestales no madereros (PFNM) en África Central para mejorar el manejo del bosque y contribuir al aumento del nivel de vida en el medio rural. Se eligieron tres sitios en la zona meridional de Camerún para analizar el impacto real de estas cadenas de PFNM sobre la economía de los hogares a mediados de los 90 y, más recientemente, en 2008-2009. Basándose en el seguimiento anual de las prácticas forestales y de los presupuestos de los hogares, el artículo muestra que los PFNM siguen siendo un recurso financieramente marginal para los hogares de estas zonas forestales. Aunque casi todos los hogares practican la recolección, muy pocos se involucran en un verdadero desarrollo comercial de estos productos. Su venta ólo supone un pequeño porcentaje de su renta global, muy por detrás de la agricultura. En este contexto, en el que los PFNM siguen siendo un simple medio de subsistencia, cualquier estrategia de promoción de estos productos tendrá que analizar detalladamente las decisiones económicas y presiones sociales que enfrentan estos hogares si aspira a ser eficaz y oportuna.

Palabras clave: productos forestales no madereros, economía, Camerún. 


\section{Introduction}

Les produits forestiers non ligneux (Pfnl) constituentils encore aujourd'hui des « ressources mineures », pour reprendre la dénomination qui lança cette thématique à la fin des années 1980 (FAO, 1990) ? Deux décennies de projets, de conférences, de publications et de politiques publiques ont manifestement changé le statut de cette panoplie souvent hétéroclite de produits forestiers autres que le bois. Rares sont aujourd'hui les programmes forestiers qui n'accordent pas une place particulière à la protection/gestion/régénération des espèces utiles aux populations locales. Au Cameroun, par exemple, une sous-direction en charge de la promotion et de la transformation des Pfnl a même été créée en 1998 au sein du ministère des Forêts.

Il en va de même chez les exploitants forestiers qui, sous la contrainte de l'aménagement forestier et/ou sous l'impulsion de la certification, intègrent maintenant les principales espèces de Pfnl dans leurs plans de gestion.

La communauté scientifique n'est pas en reste puisqu'on observe un nombre croissant de publications sur la contribution des Pfnl à la lutte contre la pauvreté (SCHRECKENBERG et al., 2006 ; Arnold, Ruiz PÉreZ, 2001). Ces travaux démontrent l'existence de marchés importants pour ces produits, à la fois à l'intérieur des frontières nationales (SHACKLETON et al., 2007) et à l'exportation (Ruiz PÉREz et al., 1999). Au Cameroun, il existe de vraies filières organisées pour plusieurs produits phares comme le njansang (Ricinodendron heudelotii), la mangue sauvage (Irvingia gabonensis), les gnetum (Gnetum spp.)... dont les chiffres d'affaires dépassent les $500000 €$ par an pour certaines d'entre elles (Ndoye, Chupezi TIEguHONG, 2004 ; AWONO et al., 2009). L'importance économique de ces filières est donc avérée à l'échelle nationale, voire internationale, en Afrique centrale.

Pourtant, l'importance substantielle des filières et des marchés de Pfnl suffit-elle pour contribuer à accroître le niveau de vie des populations rurales tout en incitant à une exploitation rationnelle et durable de ces ressources?

L'existence de marchés actifs pour certains Pfnl est évidemment une condition pour que les producteurs/collecteurs puissent en vendre davantage et accroître leurs revenus. Mais très peu de travaux indiquent aujourd'hui l'impact socio-économique réel des marchés de Pfnl sur le niveau de bien-être à l'échelle locale.
Sur la base d'enquêtes socio-économiques conduites à l'échelle villageoise en trois sites différents du Sud-Cameroun, nous tenterons de caractériser l'importance d'une certaine catégorie de Pfnl, ceux destinés à l'alimentation (photo 1). Dans son acception la plus large, le terme de Pfnl représente tous les matériaux biologiques, autres que le bois, qui sont extraits de forêts naturelles pour un usage humain. Si la notion de forêt naturelle peut paraître ambiguë, elle indique néanmoins que les Pfnl ne sont pas des produits cultivés et sont indépendants d'une régulation anthropique de l'environnement. Les animaux sauvages chassés sont le plus souvent exclus de la catégorie des Pfnl. On admet en outre généralement que les $\mathrm{Pfnl}$ sont prélevés de manière traditionnelle, avec des techniques simples qui occasionnent peu de dommages au milieu. De nombreux produits rentrent donc dans la catégorie des Pfnl, comme le montrent VAN DIJK (1999) ou LESCUYER (2000) pour la zone forestière du Cameroun. Le principal usage des Pfnl est toutefois l'alimentation.

Six variables sont retenues pour caractériser l'importance économique de l'activité de cueillette dans les économies rurales de la zone forestière du Cameroun : proportion de la population pratiquant la cueillette, les produits prélevés, les périodes de cueillette, les endroits fréquentés, les revenus générés et le niveau de consommation. L'analyse de ces résultats permettra de comprendre si les Pfnl ont perdu à cette échelle, là aussi, leur statut de ressource mineure.

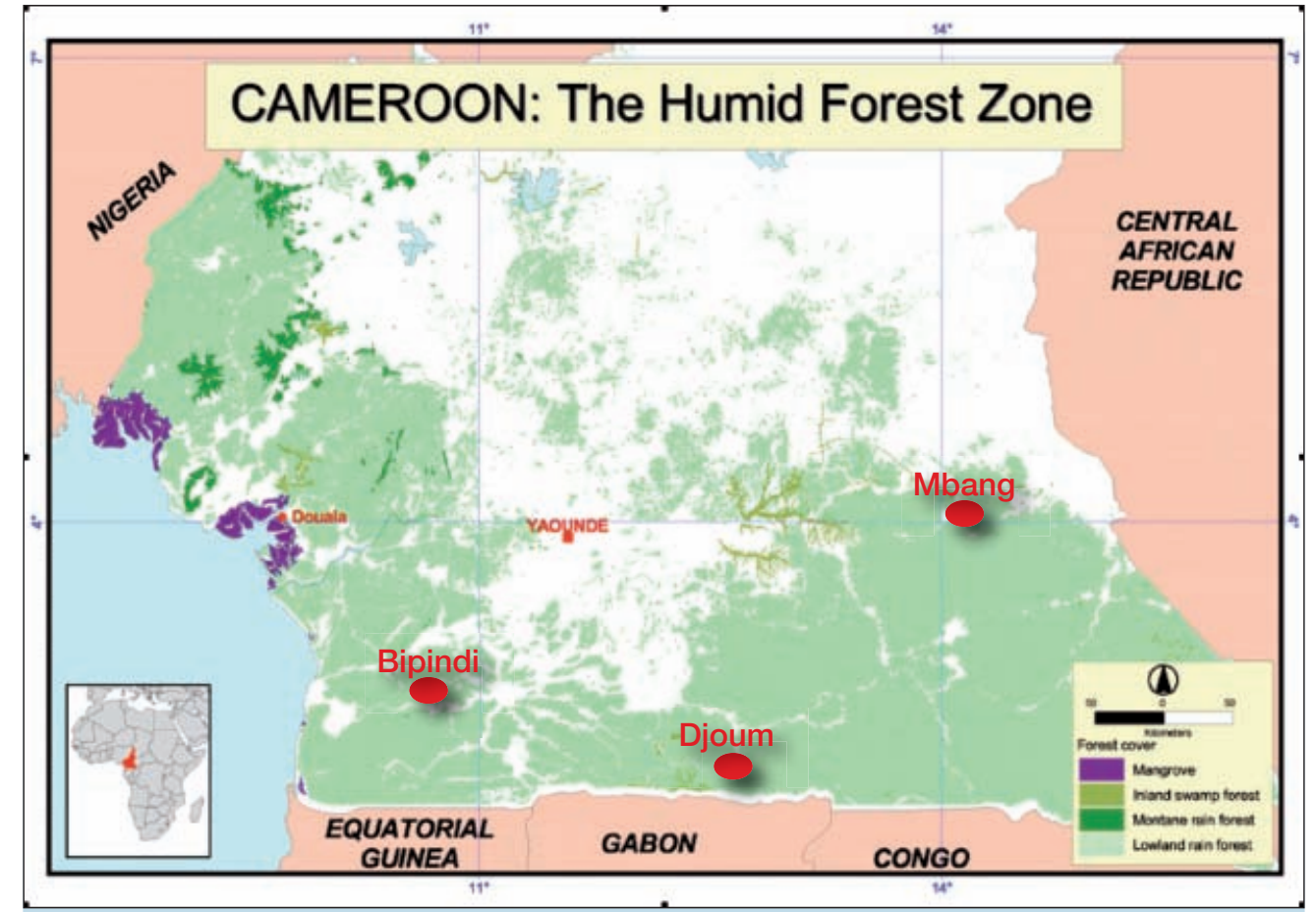

Figure 1.

Localisation des sites d'étude (source de la carte : CIFOR-IRD, 2003). 


\section{Sites et méthodes d’étude}

Trois zones ont été retenues pour analyser l'utilisation des Pfnl par les foyers en milieu rural à deux périodes, à savoir le milieu des années 1990 puis les années 2008-2009 (figure 1).

Elles sont situées dans la partie méridionale du Cameroun, très largement couverte de forêt dense. Aucun site n'a été retenu dans les deux régions du Sud-Ouest et du NordOuest du Cameroun qui sont particulièrement actives dans la production et la vente de Pfnl tels que le eru (Gnetum africanum) et le pygeum (Prunus africana) pour l'exportation respectivement vers le Nigeria (NDOYE, 2005) et l'Europe via Douala (Awono et al., 2009). La sélection des sites a davantage favorisé des zones forestières banales, où l'agriculture peu mécanisée reste dominante, où les conditions de transport sont mauvaises et où le prélèvement des produits forestiers est tourné vers les besoins de consommation avant de répondre à une potentielle demande commerciale.

Trois communes ont été retenues pour appréhender les potentielles variations géographiques pouvant exister au sein de la bande forestière camerounaise : le site de Bipindi est proche du littoral atlantique, celui de Djoum est au sud et celui de Mbang à l'est du pays. Dans chacune de ces communes, un ou plusieurs villages ont été sélectionnés pour conduire ces études relatives à l'impact socio-économique des Pfnl sur les économies rurales (tableau I).

En se reportant à la carte des marchés de Pfnl produite par RuIz PÉREz et al. (1999), les trois sites ne sont que peu concernés par ce type de commerce : aucun village n'est situé à moins de quarante kilomètres d'un marché de Pfnl et ces marchés ont de modestes chiffres d'affaires compris entre 15000 et $50000 €$ par an, toujours selon les estimations avancées par RuIz PÉREz et al. (1999). Pourtant, ces zones paraissent caractéristiques de la vaste majorité des villages localisés dans la bande forestière du Cameroun, qui font face à un degré relativement important d'enclavement.
Les communes retenues sont très largement couvertes de forêt et celle-ci est majoritairement destinée à l'exploitation du bois par le biais de concessions forestières. D'autres modes de gestion de l'espace sont également présents à Bipindi et à Djoum mais ils demeurent minoritaires. La présence de ces différents modes de gestion indique l'existence de massifs forestiers de taille et de richesse significatives, qui viennent utilement compléter la bande agroforestière où les habitants peuvent exercer librement leurs droits d'usage, dont la collecte.

Ces trois sites ont été soumis à une étude socio-économique ayant pour objet l'utilisation des Pfnl alimentaires et ayant comme unité d'analyse de base le foyer rural.

Une difficulté dans l'étude de certains Pfnl alimentaires est la variabilité de leur production naturelle, qui ne peut être appréhendée que par le suivi de ces espèces sur au moins deux années consécutives. Or, les résultats présentés ici reposent sur une base annuelle et reflètent sans doute moins une réalité moyenne que s'ils avaient porté sur une plus longue période.

Une autre difficulté vient de la définition de l'unité sociale à analyser. II est généralement admis que le foyer représente à la fois une unité de résidence, de production et de consommation (AugÉ, 1975). Il doit donc être considéré de manière plus large que la notion de ménage, qui correspond généralement à la simple famille nucléaire : si le noyau du foyer se compose en effet du mari, de sa (ses) femme(s) et de leurs enfants, d'autres personnes sont également membres à part entière du foyer. Ces différentes personnes sont le plus souvent rassemblées en une seule habitation ou en un seul groupe d'habitations voisines, ce qui vérifie le critère d'unité de résidence. Ils mangent également le plus souvent ensemble. Il est, par contre, plus difficile de définir le foyer sur la base de l'unité de production puisque les champs sont individuels, tout comme les produits et les revenus qui en sortent. Le foyer, tel qu'il est retenu dans ces travaux, est donc un groupe de personnes, parentés ou non, qui vivent et mangent ensemble sans pour autant nécessairement partager tous les moyens et fruits de leurs productions.

Tableau I.

Caractéristiques des sites d'étude.

\begin{tabular}{|c|c|c|c|c|c|}
\hline & Bipindi 1999 & Bipindi 2009 & Djoum 2008 & Mbang 1995 & Mbang 2008 \\
\hline $\begin{array}{l}\text { Marchés de Pfnl les plus } \\
\text { proches }\end{array}$ & $\begin{array}{c}\text { Kribi } 80 \mathrm{~km} \\
(C A<30000 €)\end{array}$ & $\begin{array}{c}\text { Kribi } 80 \mathrm{~km} \\
(\mathrm{CA}<30000 €)\end{array}$ & $\begin{array}{l}\text { Sangmelima } 130 \mathrm{~km} \\
\quad(C A<50000 €)\end{array}$ & $\begin{array}{l}\text { Batouri } 40 \mathrm{~km} \\
(C A<15000 €) \text {, } \\
\text { Bertoua } 80 \mathrm{~km} \\
(C A<30000 €)\end{array}$ & $\begin{array}{l}\text { Batouri } 40 \mathrm{~km} \\
(C A<15000 €) \text {, } \\
\text { Bertoua } 80 \mathrm{~km} \\
(C A<30000 €)\end{array}$ \\
\hline $\begin{array}{l}\text { Statut des forêts } \\
\text { riveraines }\end{array}$ & $\begin{array}{l}\text { Concession forestière, } \\
\text { forêt communale, parc } \\
\text { national }\end{array}$ & $\begin{array}{l}\text { Concession forestière, } \\
\text { forêt communale, parc } \\
\text { national }\end{array}$ & $\begin{array}{l}\text { Concession forestière, } \\
\text { parc national, forêt } \\
\text { communautaire }\end{array}$ & Concession forestière & Concession forestière \\
\hline Nombre de villages enquêtés & 18 & 18 & 1 & 1 & 2 \\
\hline $\begin{array}{l}\text { Nombre de foyers } \\
\text { enquêtés }\end{array}$ & $\begin{array}{c}364 \\
\text { (27\% des foyers) }\end{array}$ & $\begin{array}{c}457 \\
\text { (environ } 30 \% \text { des foyers) }\end{array}$ & $\begin{array}{c}26 \\
\text { (68 \% des foyers) }\end{array}$ & $\begin{array}{c}20 \\
\text { (100 \% des foyers) }\end{array}$ & $\begin{array}{c}64 \\
\text { (82 \% des foyers) }\end{array}$ \\
\hline Année d'enquête & 1999 & 2009 & 2008 & 1995 & 2008 \\
\hline Types d'enquête & $\begin{array}{c}\text { Diagnostic socio-écono- } \\
\text { mique : estimations } \\
\text { déclaratives }\end{array}$ & $\begin{array}{l}\text { Diagnostic socio-écono- } \\
\text { mique : estimations } \\
\text { déclaratives }\end{array}$ & $\begin{array}{l}\text { Suivi hebdomadaire des } \\
\text { prélèvements et des } \\
\text { budgets pendant } 1 \text { an }\end{array}$ & $\begin{array}{l}\text { Suivi hebdomadaire des } \\
\text { prélèvements et des } \\
\text { budgets pendant } 1 \text { an }\end{array}$ & $\begin{array}{c}\text { Suivi hebdomadaire des } \\
\text { prélèvements et des } \\
\text { budgets pendant } 1 \text { an }\end{array}$ \\
\hline
\end{tabular}


Deux méthodes ont été mises en œuvre pour réaliser ces enquêtes socio-économiques :

- Questionnaires administrés aux foyers sur la base d'un échantillonnage représentatif. Cette méthode a été utilisée dans la zone de Bipindi en 1998 sur 364 foyers et en 2009 sur 457 foyers répartis dans les 18 mêmes villages, représentant autour de $30 \%$ de la population totale de ces localités. Dans la mesure du possible, tous les membres du foyer participent au remplissage du questionnaire. C'est le type d'enquête le plus courant en matière de Pfnl (WollenberG, NAWIR, 1998), qui consiste à récapituler sur une base annuelle les pratiques et les produits collectés, sans observation directe. Il présente l'avantage d'être facile à conduire sur un grand nombre de villages, mais les données résultent d'une estimation faite par le foyer, qui est souvent biaisée. AmBRoSE-0رI (2003) montre que les foyers ont tendance à surestimer les revenus annuels tirés des Pfnl, en se focalisant sur les sommes importantes d'argent qui rentrent en une fois plutôt que sur la série de petits revenus qui sont étalés sur l'année.

- Suivi annuel des prélèvements en forêt (nom du cueilleur, espèce, nombre de pièces, endroit, destination, technique, durée...) et des budgets des foyers des villages sélectionnés. Cette approche utilise deux supports d'enquête, l'un portant sur les prélèvements effectués en forêt par tous les membres du foyer et l'autre sur les revenus et dépenses occasionnés par semaine dans le foyer. Ces deux fiches de suivi sont administrées sur une base hebdomadaire pendant une année complète dans la majorité des foyers du village, auprès des principaux membres de chaque foyer. En dehors de son côté exhaustif, cette démarche permet de trianguler partiellement l'information puisqu'une partie des données relevées dans les questionnaires « prélèvement » va se retrouver dans les fiches «budget » - tous les Pfnl commercialisés, par exemple. Il en est de même avec les fiches "budget " puisque ce qui est vendu dans un foyer doit se retrouver acheté par un autre foyer quand il s'agit de commerce intra-villageois. Ce type d'enquête a été conduit à Djoum en 2008 et à Mbang en 1995 puis 2008 dans plusieurs dizaines de foyers (cf. tableau I). En raison de sa lourdeur, ce type d'enquête détaillée ne peut être réalisé que dans un nombre réduit de villages. Pourtant, si le nombre de villages est restreint, ces enquêtes ont été administrées auprès de la plupart des foyers résidents, couvrant entre $68 \%$ et $100 \%$ des familles implantées dans ces villages.
Les données présentées dans cet article résultent de la mise en œuvre de ces deux approches. Une interrogation se pose sur leur comparabilité puisque, si les informations recherchées étaient identiques, les protocoles d'enquête ont divergé. Deux éléments permettent toutefois de réduire la portée de cette limite méthodologique. D’une part, les protocoles d'enquête ont été les mêmes dans le temps sur chacun des terrains, à savoir le diagnostic a posteriori à Bipindi en 1999 et en 2009 tandis qu'un suivi hebdomadaire a été organisé à Mbang en 1995 et en 2008. La comparaison temporelle des résultats de chacun des sites d'étude est donc sans équivoque. D'autre part, les deux méthodes indiquent des tendances similaires, en matière d'évolution de l'activité de cueillette et de développement économique, même si les données tirées des suivis hebdomadaires sont plus fiables et détaillées.

\section{Proportion de la population pratiquant la cueillette dans les zones d'étude}

Dans les trois sites d'étude, une très large majorité des individus pratiquent l'activité de cueillette, au moins de manière ponctuelle, comme l'indique le tableau II.

Le moindre score obtenu à Bipindi s'explique sans doute par une population plus largement urbanisée et fonctionnarisée que dans les deux autres sites. Cette frange de la population tend à voir la cueillette comme une activité mineure, plutôt négativement connotée puisque pratiquée par ceux qui n'ont pas la compétence de trouver un « job » ou le courage d'entretenir leurs champs. Dans une moindre mesure, on entend aussi ce discours dans les villages pour les personnes qui passent un temps jugé démesuré en forêt à ramasser ce que livre la nature plutôt que de s'employer au « travail de la machette».

La collecte des Pfnl demeure une activité très largement pratiquée, ce qui indique le maintien dans ces sociétés d'une relation forte entre l'écosystème et la population. Toutefois, si presque tous les foyers pratiquent la cueillette, tous ne sont toutefois pas impliqués dans la vente des Pfnl. Que ce soit sur le site de Bipindi ou de Mbang, on observe une décroissance de la proportion de foyers vendant des Pfnl. En outre, comme précisé plus loin, les revenus tirés de cette activité demeurent faibles pour la très grande majorité de ces foyers : il s'agit le plus souvent d'un commerce ponctuel voire anecdotique.

Tableau II.

Nombre de foyers pratiquant la cueillette.

\begin{tabular}{|c|c|c|c|c|c|}
\hline & Bipindi 1999 & Bipindi 2009 & Djoum 2008 & Mbang 1995 & Mbang 2008 \\
\hline Nombre de villages échantillonnés & 18 & 18 & 1 & 1 & 2 \\
\hline Nombre de foyers extrayant les Pfnl & $298(81 \%)$ & $402(88 \%)$ & $25(96 \%)$ & $20(100 \%)$ & $63(98 \%)$ \\
\hline Nombre de foyers vendant les Pfnl & $144(39 \%)$ & $113(29 \%)$ & $25(96 \%)$ & $20(100 \%)$ & $43(67 \%)$ \\
\hline
\end{tabular}




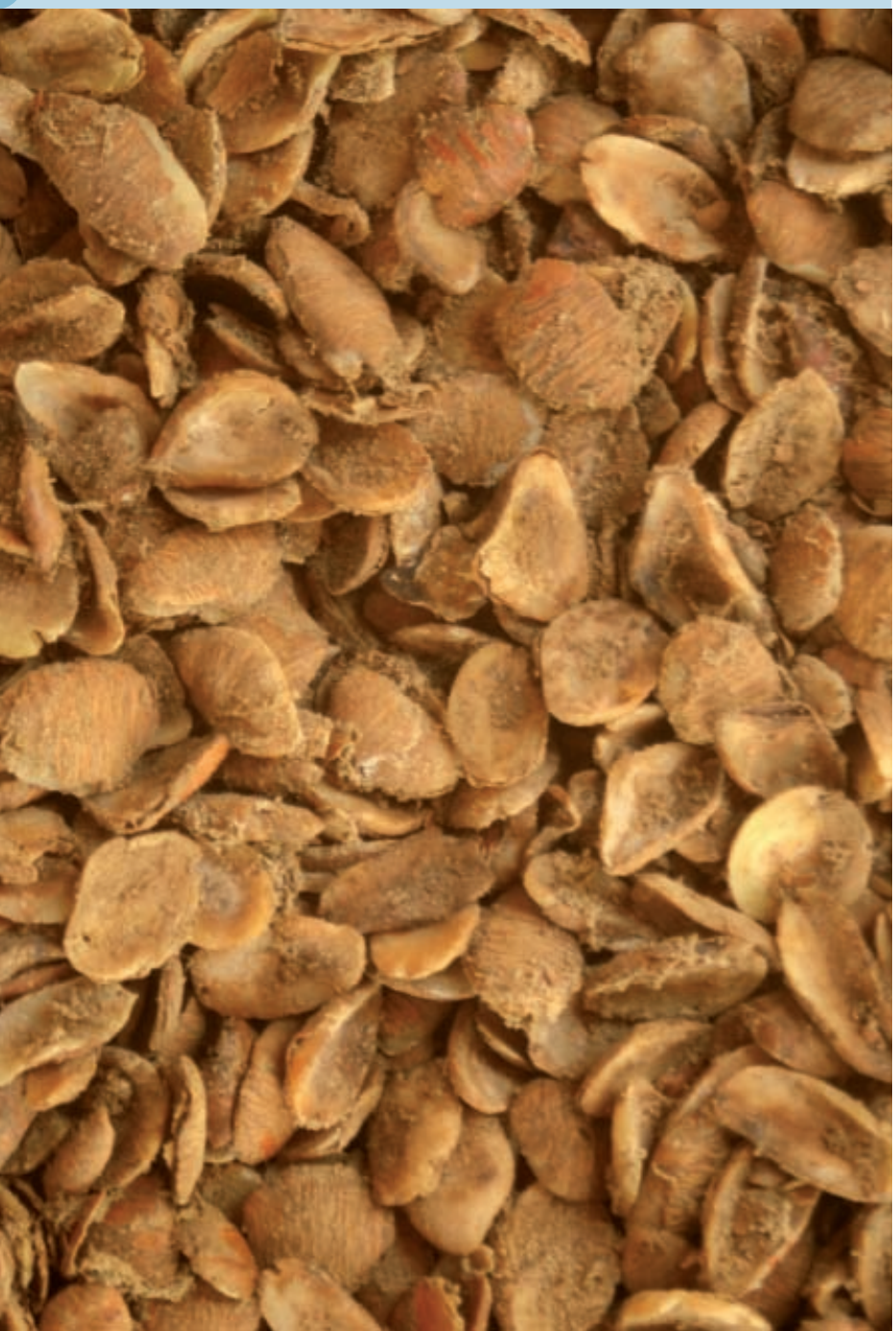

Photo 2.

Amande de mangue sauvage, Irvingia gabonensis. Photo G. Lescuyer.
De nombreux critères peuvent être utilisés pour identifier les espèces de Pfnl les plus couramment utilisées à l'échelle locale. Sont à retenir le plus souvent les espèces qui produisent un volume important et/ou celles qui peuvent faire l'objet de commercialisation. C'est manifestement ce type de critère qui prédomine dans les déclarations des foyers interrogés à Bipindi, qui placent la mangue sauvage et les noix de palme aux premiers rangs (tableau III, photo 2).

Pourtant, si on analyse les actes de prélèvement selon les suivis annuels à Mbang et à Djoum, ce sont d'autres espèces de Pfnl qui apparaissent comme étant les plus utilisées : champignons (Pleurotidae spp...), escargots (Actina spp...), palmiers (Elaeis guineensis, Raphia monbuttorum, Raphia hookeri). Ces espèces plus communes constituent en fait l'essentiel de la pratique de cueillette à l'échelle locale, pour plusieurs raisons : elles sont présentes presque toute l'année, abondantes, appréciées et le plus souvent disponibles aux abords du village.

Il paraît donc étonnant que si peu d'attention soit aujourd'hui accordée par les organisations de promotion des Pfnl aux espèces de champignon et d'escargot ainsi qu'au vin " traditionnel " tiré du palmier raphia ou du palmier à huile. Une des raisons tient sans doute à la distribution spatialement aléatoire de la production d'escargots et de champignons. Une telle incertitude n'existe pas pour la production de vin traditionnel (photo 3), dont la promotion est rarement faite par les organisations d'appui, en dépit de son importance locale et des marchés urbains demandeurs.

\section{Périodes et sites de cueillette}

Généralement, la grande saison pluvieuse au Cameroun est à considérer comme la principale période de fructification de nombreuses espèces qui produisent des Pfnl, comme la mangue sauvage, le njansan... (EYog Matıg et al., 2006). C'est à cette période que la cueillette devient une activité à part entière puisque les familles organisent des « sorties de cueillette » en forêt afin de ramasser un volume important de ces produits qui seront ensuite partiellement vendus. La

Tableau III.

Principales espèces utilisées.

\begin{tabular}{|c|c|c|c|c|c|}
\hline & Bipindi 1999 & Bipindi 2009 & Djoum 2008 & Mbang 1995 & Mbang 2008 \\
\hline $\begin{array}{l}\text { Nombre d'espèces } \\
\text { mentionnées }\end{array}$ & 48 & & 48 & 119 & 76 \\
\hline \multirow[t]{4}{*}{$\begin{array}{l}\text { Principales espèces } \\
\text { mentionnées ou utilisées }\end{array}$} & $\begin{array}{l}\text { Ndo'o (Irvingia } \\
\text { gabonensis) }\end{array}$ & Palmier à huile & $\begin{array}{l}\text { Palmier à huile } \\
\text { et raphia }\end{array}$ & Escargots (petits) & Escargots \\
\hline & $\begin{array}{l}\text { Palmier à huile (Elaeis } \\
\text { guineensis) }\end{array}$ & Ekouk & Vers blancs & Champignons (petits) & Champignons \\
\hline & Noisettes (Coula edulis) & Ndo'o & Champignons & $\begin{array}{c}\text { Doko (Irvingia } \\
\text { gabonensis) }\end{array}$ & $\begin{array}{l}\text { Ndjansan (Ricino - } \\
\text { dendron heudelotii) }\end{array}$ \\
\hline & Champignons & Essok (Garcinia lucida) & Chenilles & $\begin{array}{l}\text { Igname sauvage } \\
\text { (Dioscorea spp.) }\end{array}$ & $\begin{array}{l}\text { Doko (Irvingia } \\
\text { gabonensis) }\end{array}$ \\
\hline
\end{tabular}


figure 2 montre la fréquence des actes de cueillette dans les trois sites d'étude sur une base annuelle. À Bipindi, sur la base de déclarations des enquêtés, la grande saison humide - comprise entre août et octobre - apparaît comme cruciale pour cette activité.

Le calendrier de cueillette présente un profil tout à fait différent à Mbang en 2008, marqué par une activité importante au moment de la petite saison pluvieuse en mars-juin. Deux raisons semblent expliquer ce calendrier. D'une part, la petite saison pluvieuse est la plus propice à l'apparition des escargots et des champignons, qui sont fortement recherchés à Mbang. D’autre part, l'année 2008 semble avoir été une année basse pour la production des mangues sauvages, ce qui a entraîné un faible nombre de sorties de cueillette en forêt pour les mois de septembre et octobre.

Le calendrier de cueillette établi pour Djoum en 2008 paraît un compromis entre ceux de Bipindi et de Mbang. Il est marqué par deux saisons d'intense cueillette, autour d'avril et

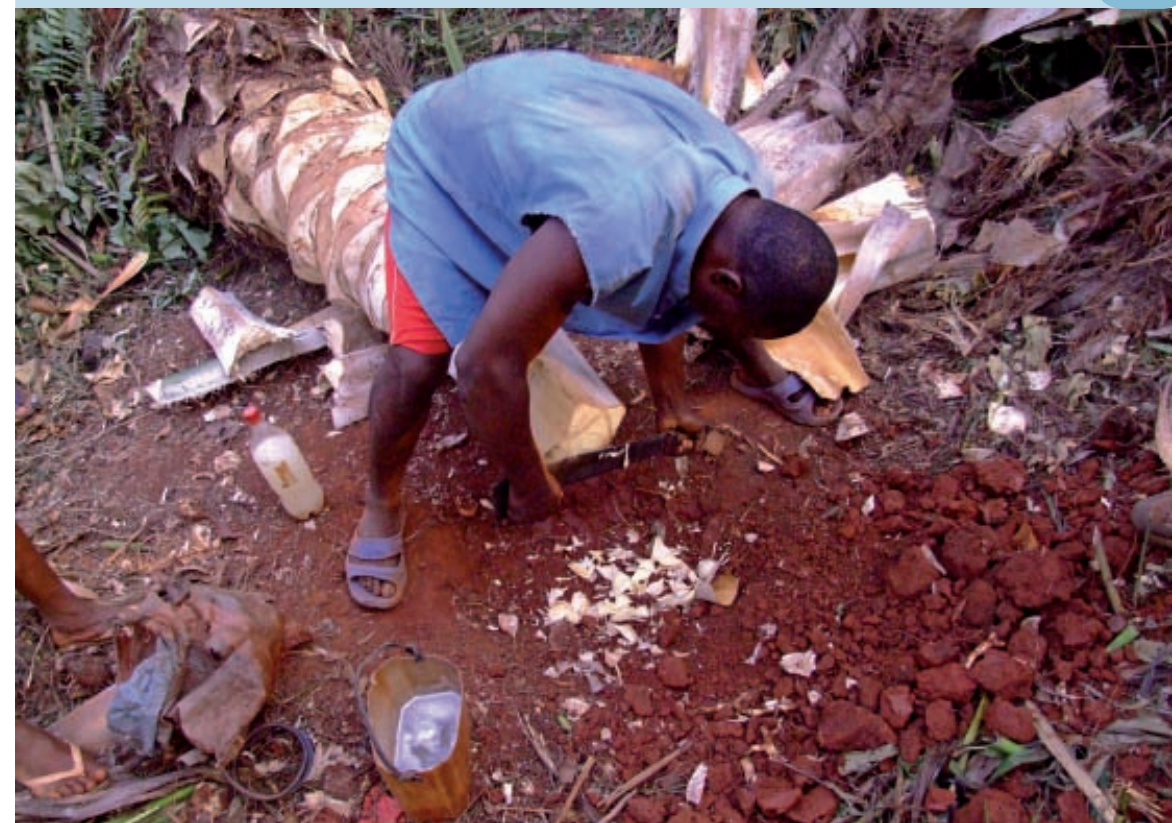

Photo 3.

Extraction du vin de palme.

Photo V. Toison.

autour de septembre, qui complètent une cueil-

lette quotidienne et proche du village tout au long de l'année, notamment pour s'approvisionner en vin de palme. À Djoum, comme à Mbang, la petite saison des pluies apparaît comme une période importante de cueillette, ce qui n'était pas le cas pour Bipindi : plutôt que des différences de pratiques, il est probable que la technique d'enquête employée à Bipindi tende à favoriser une seule importante période de cueillette, celle de ramassage des mangues sauvages, qui marque l'esprit des personnes interrogées.

L'étude des déplacements des cueilleurs dans les sites de Mbang et de Djoum montre que la forêt « primaire » constitue une source mineure de Pfnl (figure 3).

Cette activité a lieu essentiellement dans les espaces fortement dégradés comme les agroforêts et le « domaine forestier non permanent ». Comme le montrent d'autres travaux au Cameroun (VAN DIJK, 1999 ; AMBrose-OJI, 2003 ; SonwA et al., 2007), il existe une corrélation positive entre le nombre d'espèces de Pfnl présentes dans un milieu et son degré de perturbation anthropique. Cela a deux conséquences. D'une part, la promotion des Pfnl n'est qu'un moyen très partiel pour inciter à une meilleure gestion des forêts naturelles par les populations locales, puisque la plupart de ces produits ne proviennent pas de forêts non perturbées. D'autre part, la cueillette est une activité qui est combinée avec d'autres systèmes de production, comme l'agriculture ou la chasse, ce qui appelle à une vision intégrée de ces pratiques (ARnold, Ruiz Pérez, 2001).

Une première étape dans l'analyse intégrée de ces pratiques locales d'exploitation de la nature est d'expliciter leur poids économique relatif dans les budgets des ménages et, au-delà, dans ces économies rurales.

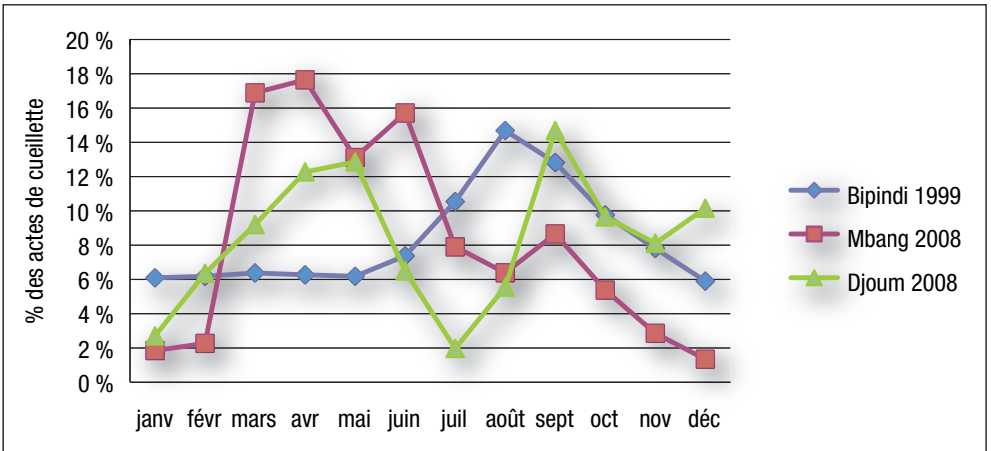

Figure 2.

Fréquences intra-annuelles des actes de cueillette.

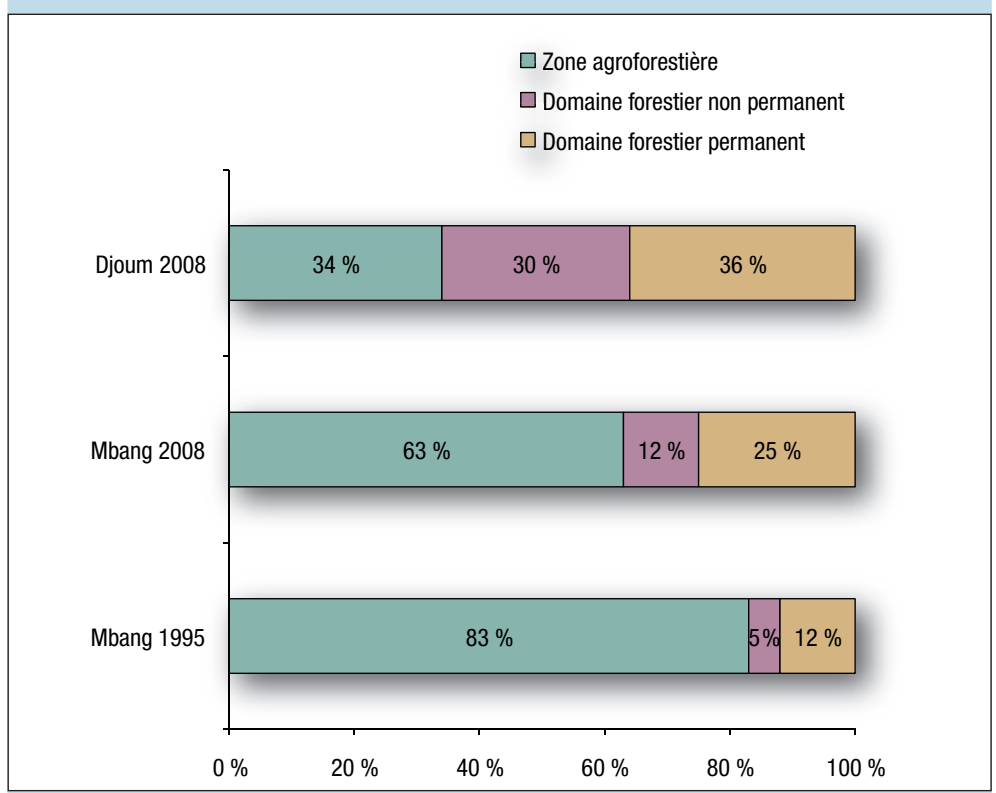

Figure 3.

Types et fréquentation des espaces de cueillette. 


\section{Revenus et valeur économique des Pfnl}

Quelle que soit la méthode retenue - estimation a posteriori des revenus annuels ou suivi hebdomadaire des revenus - l'activité de cueillette apparaît comme une source secondaire de revenus pour les foyers des trois zones étudiées (tableau IV). Elle contribue au mieux à $8 \%$ des revenus à Bipindi en 1999, une zone où des commerçants spécialisés en Pfnl sont installés, ce qui relève le niveau moyen. Dans tous les autres cas, le revenu brut tiré de l'activité de cueillette représente entre $2 \%$ et $4 \%$ du revenu total moyen des foyers ruraux, une partie importante étant assurée par la vente sous forme brute ou distillée des vins de palme et de raphia.

Dans tous les cas, les revenus de la cueillette demeurent très largement inférieurs à ceux de l'agriculture ou résultant d'activités salariées. Étant donné ce très faible impact des Pfnl sur les finances des foyers, la promotion de ces filières a peu de chances d'avoir un impact significatif sur les niveaux de vie : même en triplant les volumes actuellement vendus, le revenu généré s'établira seulement entre $5 \%$ et $10 \%$ du revenu total du foyer. À titre comparatif, une simple augmentation de $10 \%$ du prix du plantain ou du cacao aura un effet beaucoup plus sensible sur les pratiques villageoises que toute initiative, même ambitieuse, de valorisation des Pfnl.

L'amélioration remarquable du niveau de revenu à Mbang et à Bipindi entre 1995-1999 et 2008-2009, liée principalement au développement de l'agriculture, ne change rien à ce constat : bien qu'il se vende plus de Pfnl aujourd'hui qu'il y a dix ans les revenus tirés de la cueillette ont significativement augmenté en valeur absolue -, la part de cette activité dans le revenu total des foyers s'est stabilisée à un niveau bas ou s'est dégradée. L'accroissement des revenus villageois n'a donc pas profité à cette activité et, selon un schéma bien connu (ARNOLD, RUIz PÉREZ, 2001), il est mis en évidence que certains produits manufacturés se sont substitués à certains Pfnl. L'exemple du « cube Maggi », qui parfume le moindre bouillon au village, est le plus édifiant : il remplace aujourd'hui toute une série de Pfnl " aromatisants » qui demeurent connus par les usagers mais qui ne sont plus prélevés, comme le montre le dernier inventaire des espèces de Pfnl réalisé à Mbang (tableau III).
Toutefois, la contribution des Pfnl au niveau de vie des populations rurales ne se traduit que partiellement par les revenus qu'ils octroient (photo 4).

Dans la partie méridionale du Cameroun, la plupart de ces ressources sont prélevées pour être autoconsommées ou échangées localement sans l'intermédiaire du marché. Leur valeur économique ne correspond donc pas à leur valeur financière, c'est-à-dire la sommation des revenus qu'ils génèrent. Elle peut pourtant être estimée à partir du volume total prélevé de Pfnl, qui est ensuite multiplié par les prix de chacun de ces produits sur un marché proche, pour autant que ce marché soit concurrentiel (LESCUYER, 2000). Cette dernière condition est plus ou moins respectée pour les trois zones échantillonnées.

Comme le montre le tableau V, les revenus tirés de la cueillette ne représentent qu'une faible part des bénéfices globalement générés par l'usage des Pfnl au sein du foyer. Au mieux, comme à Mbang ou à Bipindi en 2008-2009, les revenus composent environ un cinquième de la valeur économique. Au pire, ces revenus sont négligeables par rapport aux bénéfices liés à leur consommation, comme à Mbang en 1995. Dans ces économies encore peu insérées dans les réseaux commerciaux, les Pfnl constituent avant tout des éléments de subsistance, ce qui n'incite ni à la domestication de ces produits, ni à une amélioration de leur commercialisation, les efforts déployés pour cela pouvant coûter plus cher que les bénéfices attendus (BELCHER et al., 2005).

En outre, l'exemple de Mbang, avec l'augmentation significative des revenus tirés des Pfnl entre 1995 et 2008 et la diminution parallèle de la valeur économique globale de ces produits, donne à penser qu'avec l'augmentation relative du niveau de vie et l'ouverture plus grande de ces villages aux circuits marchands les produits de la cueillette seraient aujourd'hui plus souvent monétarisés ; mais qu'en contrepartie cette activité se focaliserait sur un nombre restreint d'espèces, comme l'indique le tableau III. Bref, les Pfnl seraient cueillis plus souvent pour vendre, ce qui réduirait année après année le spectre des produits qui sont ramassés et pourrait éroder à terme les savoirs qui y sont associés.

Tableau IV.

Revenus moyens annuels par foyer.

\begin{tabular}{|c|c|c|c|c|c|c|c|c|c|c|}
\hline \multirow[b]{3}{*}{ Agriculture } & \multicolumn{2}{|c|}{ Bipindi 1999} & \multicolumn{2}{|c|}{ Bipindi 2009} & \multicolumn{2}{|c|}{ Djoum 2008} & \multicolumn{2}{|c|}{ Mbang 1995} & \multicolumn{2}{|c|}{ Mbang 2008} \\
\hline & Fcfa & $\%$ & Fcfa & $\%$ & Fcfa & $\%$ & Fcfa & $\%$ & Fcfa & $\%$ \\
\hline & 147308 & 31 & 644254 & 53 & 956332 & 64 & 143237 & 61 & 288104 & 45 \\
\hline Chasse & 24990 & 5 & 25714 & 2 & 34960 & 2 & 8563 & 4 & 31145 & 5 \\
\hline Cueillette & 37580 & 8 & 47242 & 4 & 33940 & 2 & 3949 & 2 & 8866 & 2 \\
\hline Pêche & 6202 & 1 & & & 2633 & 0 & 7380 & 3 & 61593 & 10 \\
\hline Dons, épargne & 25385 & 5 & & & 113740 & 8 & 24712 & 10 & 15570 & 2 \\
\hline Salaires & 70805 & 15 & 293306 & 24 & 72833 & 5 & 37084 & 16 & 155745 & 24 \\
\hline Pensions & 34658 & 7 & 33514 & 3 & 0 & 0 & 0 & 0 & 0 & 0 \\
\hline Autres revenus & 123290 & 26 & 168376 & 14 & 289347 & 19 & 9208 & 4 & 74461 & 12 \\
\hline Revenu total & 470218 & & 1212406 & & 1503786 & & 234133 & & 635481 & \\
\hline
\end{tabular}




\section{Adapter les stratégies de promotion des Pfnl au contexte}

Généralement, à la promotion des Pfnl s'accordent deux objectifs principaux : celui de contribuer à une gestion plus diversifiée des ressources forestières et celui de participer à l'accroissement des niveaux de vie en milieu rural. Qu'en est-il réellement au regard des résultats précédemment présentés?

Toutes les recherches conduites dans la zone forestière du Cameroun sur ce sujet indiquent que la forêt primaire reste une source secondaire de Pfnl. L'essentiel des prélèvements a lieu dans des zones fortement anthropisées. Il n'en demeure pas moins vrai que certaines essences forestières prisées pour leurs Pfnl, comme le moabi (Baillonella toxisperma), sont généralement localisées loin des villages, parfois à l'intérieur de concessions forestières, ce qui peut engendrer un conflit entre populations et exploitant. Mais, d'une part, ces ressources sont très peu nombreuses au regard du large spectre des Pfnl couramment utilisés et, d'autre part, des mesures relativement simples de gestion peuvent être prises pour éviter ou limiter ce type de conflit (ASSENG ZE, 2008).

Un grand nombre de raisons sont évoquées dans la littérature pour expliquer le faible impact de la commercialisation des Pfnl sur les économies locales dans la zone forestière méridionale du Cameroun (TCHATAT et al., 1995 ; AMBROSE-OJI, 2003 ; VAN DIJK et al., 2003) : prix volatils, transport peu sûr et irrégulier, communication difficile, connotation socialement négative de l'activité de cueillette, production irrégulière des ressources, faible transformation, manque d'accès au crédit... (photo 5).

Ces obstacles manifestes font de la cueillette de Pfnl une activité peu porteuse de développement économique, même si elle contribue à un niveau de vie minimal des populations en zones rurales. Contrairement à d'autres parties du Cameroun, comme les régions du Nord-Ouest et du Sud-Ouest, la plupart des Pfnl de la zone forestière méridionale sont utilisés selon une stratégie de subsistance par les populations, avant tout pour leur autoconsommation et éventuellement pour un revenu qui reste marginal. Ce n'est ni une stratégie de revenu supplémentaire, où l'exploitation est orientée pour répondre à une demande marchande, ni une stratégie de gestion intégrée, où les Pfnl sont domestiqués et intègrent le cycle agricole, pour reprendre les catégories proposées par BELCHER et al. (2005).

Cette erreur courante d'analyse génère pour le Sud-Cameroun des recommandations inadaptées en termes de tenure forestière/foncière, d'accès à l'information, d'appui organisationnel... À l'heure actuelle, et étant donné cette stratégie de subsistance s'appliquant aux Pfnl dans les économies rurales, il est possible de s'interroger sur la légitimité d'une politique

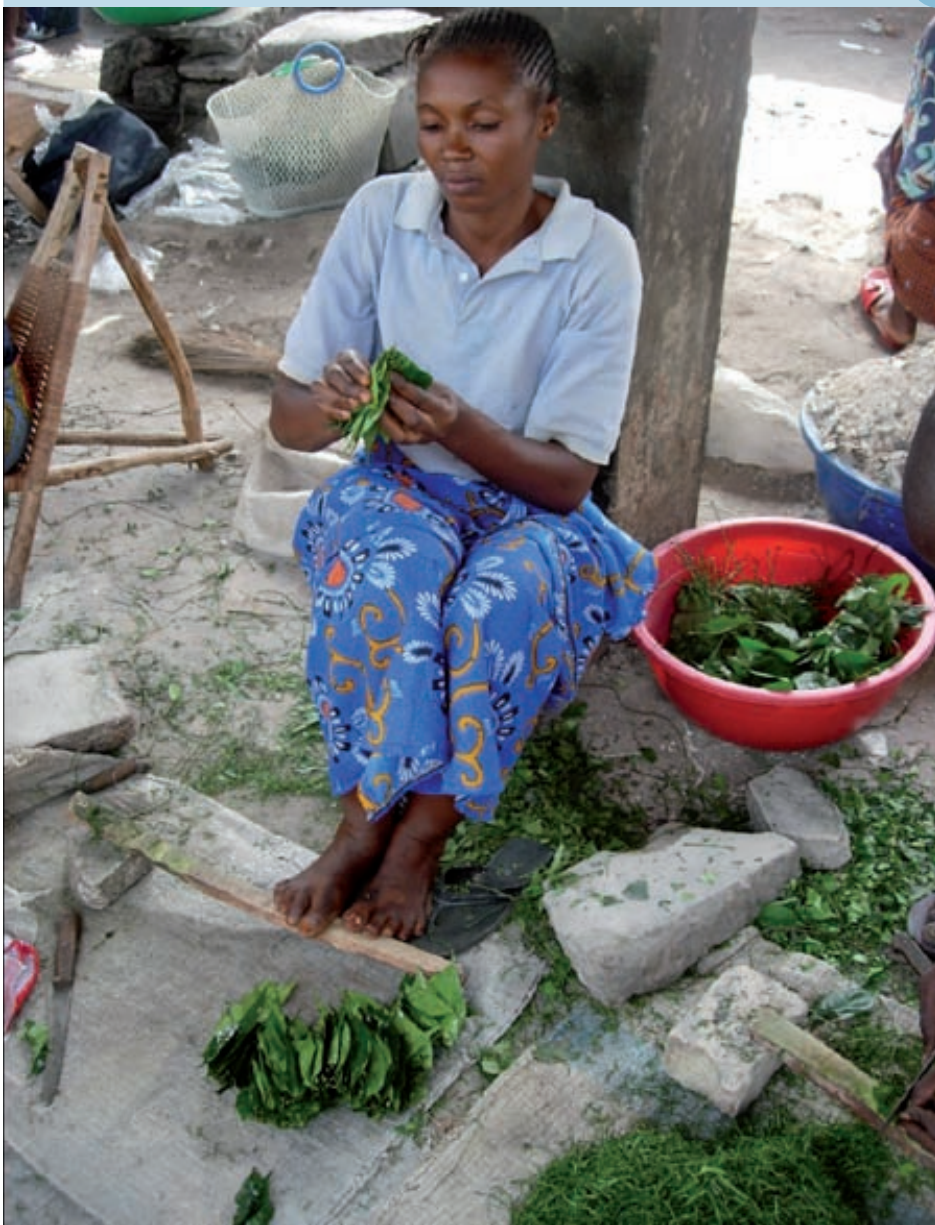

Photo 4.

Une vendeuse de produits forestiers non ligneux. Photo A. Awono.

de développement des Pfnl dans la zone forestière du SudCameroun : elle présente le risque de détourner une partie du temps de travail des populations rurales vers une activité qui accroîtra au mieux de quelques pourcents leurs revenus au détriment d'activités plus lucratives et moins incertaines, comme les plantations agricoles ou l'abattage du bois. Faciliter le développement économique de la cueillette tout en évitant d'enfermer ces populations dans un nouveau piège à pauvreté (Arnold, RuIz PÉREZ, 2001) requiert aujourd'hui d'intégrer cette activité dans une compréhension plus globale de l'usage des espaces et des ressources à l'échelle locale, reposant notamment sur une analyse fine de leurs contraintes temporelles et sociales ainsi que de leurs arbitrages économiques.

Tableau V.

Valeur économique des Pfnl (en francs Cfa par foyer et par an).

\begin{tabular}{|l|r|r|r|r|}
\hline & Djoum 2008 & Bipindi 2009 & Mbang 1995 & \\
\hline Revenus financiers & & & \\
\hline Valeur économique des prélèvements & 33940 & 47242 & 3949 & 8608 \\
\hline
\end{tabular}




\section{Remerciements}

L'auteur tient à remercier Émilie Perge, doctorante à l'Université du Sussex, qui a mis à sa disposition les résultats de son enquête socio-économique réalisée en 2009 dans les villages autour de Bipindi, grâce à un financement de la fondation Tropenbos International. Ces résultats ont également bénéficié d'un soutien financier du programme "Agriculture et Développement Durable" de l'Agence Nationale de la Recherche, par le biais du projet Popular.

\section{Références bibliographiques}

AMBROSE-OJI B., 2003. The contribution of NTFPs to the livelihoods of the "forest poor» : Evidence from the tropical forest zone of South-West Cameroon. International Forestry Review, 5 (2) : 106-17.

ARNOLD J. E. M., RUIZ PÉREZ M., 2001. Can non-timber forest products match tropical forest conservation and development objectives ? Ecological Economics, 39 : 437-447.

ASSENG ZE A., 2008. Gestion durable des produits forestiers non ligneux dans la concession forestière de Pallisco. Rome, Italie, Fao, Étude pilote sur les techniques d'exploitation forestière, $n^{\circ} 24,40$ p.

AUGÉ M., 1975. Les domaines de la parenté. Paris, France, Maspero, 288 p.

AWONO A., MANIRAKIZA D., INGRAM V., 2009. Étude de base de la filière Ndo'o (Irvingia spp.) dans les provinces du Centre, du Sud et du littoral du Cameroun. Yaoundé, Cameroun, Fao-Cifor-Snv-Icraf-Comifac, projet Gcp/Raf/408/EC, 100 p. BELCHER B., RUIZ PÉREZ M., ACHDIAWAN R., 2005. Global patterns and trends in the use and management of commercial NTFPs : Implications for livelihoods and conservation. World Development, 33 (9) : 1435-1452.

CIFOR-IRD, 2003. Cameroon's forests and people. Bogor, Indonésie, Cifor, cédérom.

EYOG MATIG O., NDOYE O., KENGUE J., AWONO A., 2006. Les fruitiers forestiers comestibles du Cameroun. Cotonou, Bénin, Ipgri, Regional Office for West and Central Africa, 204 p.

FAO, 1990. The major significance of " minor » forest products. The local use and value of forest in the West African humid forest zone. Rome, Italie, Fao, Community Forestry Note $n^{\circ}$ 6, $106 \mathrm{p}$.

LESCUYER G., 2000. Évaluation économique et gestion viable de la forêt tropicale. Réflexion sur un mode de coordination des usages d'une forêt de l'Est-Cameroun. Thèse, École des hautes études en sciences sociales, Paris, France, 417 p.

NDOYE O., 2005. Commercial issues related to non-timber forest products. In : Pfund J. L., Robinson P. (éd.). Non-timber forest products between poverty alleviation and market forces. Berne, Suisse, Intercooperation, p. 14-19.

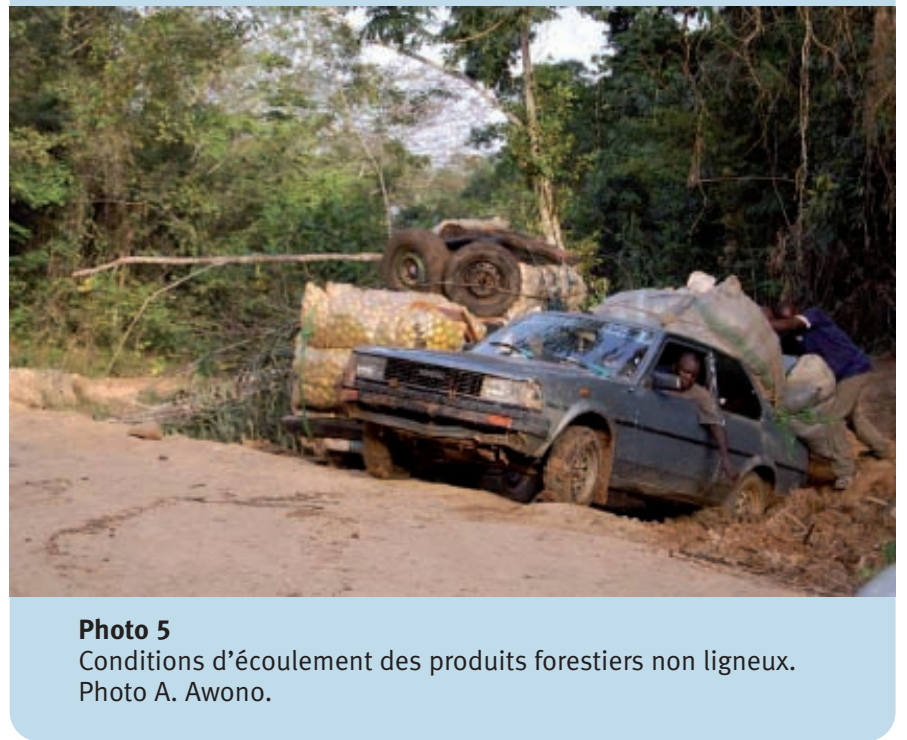

NDOYE O., CHUPEZI TIEGUHONG J., 2004. Forest resources and rural livelihoods : The conflict between timber and nontimber forest products in the Congo Basin. Scandinavian Journal of Forest Resources, 19 (4) : 36-44.

RUIZ PÉREZ M., NDOYE O., EYEBE A., 1999. Marketing of nonwood forest products in the humid forest zone of Cameroon. Unasylva, 198 : 12-19.

SCHACKLETON S., SHANLEY P., NDOYE O., 2007. Invisible but viable : recognising local markets for non-timber forest products. International Forestry Review, 9 (3) : 697-712.

SCHRECKENBERG K., AWONO A., DEGRANDE A., MBOSSO C., NDOYE O., TCHOUNDJEU Z., 2006. Domesticating indigenous fruit trees to reduce poverty. Forests, Trees and Livelihoods, $16: 35-51$.

SONWA D. J., NKONGMENECK B. A., WEISE S. F., TCHATAT M., ADESINA A. A., JANSSENS M. J. J., 2007. Diversity of plants in cocoa agroforests in the humid forest zone of Southern Cameroon. Biodiversity Conservation, 16 : 2385-2400.

TCHATAT M., PUIG H., TIKI MANGA T., 1995. Les jardins de case des provinces du centre et du sud du Cameroun : description et utilisation d'un système agroforestier traditionnel. Journal d'Agriculture Traditionnelle et de Botanique Appliquée, 37 (2) : 165-182.

VAN DIJK J. F. W., 1999. Non-timber forest products in the Bipindi-Akom II Region, Cameroon. A socio-economic and ecological assessment. Université de Wageningen, Pays-Bas, Tropenbos-Cameroon Series 1, $197 \mathrm{p}$.

VAN DIJK J. F. W., ONGUENE N. A., KUYPER T. W., 2003. Knowledge and utilization of edible mushrooms by local populations of the rain forest of south Cameroon. Ambio, 32 (1) : 19-23.

WOLLENBERG E., NAWIR A. S., 1998. Estimating the incomes of people who depend on forests. In : Wollenberg E., Ingles A. (éd.). Income from the forest. Methods for the development and conservation of forest products for local communities. Bogor, Indonésie, Cifor, p. 157-187. 